\title{
ESCLEROCRONOLOGÍA EN VALVAS DE MYTILUS SPP: ANÁLISIS DEL SITIO CCH4 (PARQUE NACIONAL MONTE LEÓN, SANTA CRUZ, ARGENTINA) E IMPLICACIONES PARA LA ARQUEOLOGÍA DE PATAGONIA
}

PATRICIA A. LOBBIA

\section{RESUMEN}

Los restos malacológicos constituyen una parte abundante del registro arqueológico en la costa patagónica. Estos depósitos brindan información acerca de la ocupación humana del litoral atlántico de Patagonia mostrando que constituyeron un recurso que ha sido explotado al menos desde hace 7.400 años AP. Considerando que se trata de un recurso disponible todo el año, es fundamental determinar la estación en la que fueron recolectados ya que esta información no solo permitiría dilucidar la importancia del recurso en la economía de los grupos humanos involucrados, sino también ofrecería información valiosa para el análisis de la ocupación humana en las costas. El objetivo de este trabajo consiste en determinar la estacionalidad de recolección de los mejillones Mytilus spp. provenientes del sitio CCH4, P.N. Monte León. La muestra analizada consiste en valvas de estos moluscos que fueron acondicionadas para su análisis esclerocronológico. Se analizó el patrón de crecimiento de las valvas, se calcularon incrementos máximos y mínimos y se compararon los resultados obtenidos con datos de moluscos actuales analizados por otros investigadores. Los resultados obtenidos demostraron que el mayor porcentaje (42,30\%) de moluscos fueron recolectados en verano. En invierno se recolectó el 30,77\% de los mejillones y valores menores fueron observados en otoño (15,28\%) y primavera (11,54\%). Esto estaría indicando, por un lado, una utilización del recurso durante todo el año y también una variación en la intensidad de la actividad de recolección de acuerdo a la estación.

PALABRAS CLAVE: Mytilus spp., zooarqueología, esclerocronología, Patagonia.

Laboratorio de Zooarqueología y Tafonomía de Zonas Áridas, IDACOR-CONICET, Museo de Antropología, Facultad de Filosofía y Humanidades, Universidad Nacional de Córdoba. Avenida Hipólito Yrigoyen 174, (5000) Córdoba, Argentina. E-mail: plobbia@ffyh.unc.edu.ar. 


\title{
SCLEROCHRONOLOGY IN MYTILUS SPP.: ANALYSIS OF SITE CCH4, (MONTE LEON NATIONAL PARK, SANTA CRUZ, ARGENTINA) AND IMPLICATIONS FOR THE ARCHAEOLOGY OF PATAGONIA
}

\begin{abstract}
Malacological remains constitute an abundant part of the archaeological record in the Patagonian coast. These deposits provide information about human occupation of the Atlantic coast of Patagonia showing that it is a resource that has been exploited at least, since 7.400 years BP. Being a resource available all year, it is essential to determine the season in which they were collected, this information will not only provide information about the importance of the resource in the economy of the human groups, but also provide valuable information for analyzing human occupation on the coast. The aim of this study is to determine the seasonal harvesting of Mytilus spp. from the site CCH4, N.P. Monte León. The sample analyzed consists of shells of Mytilus spp. that were conditioned for sclerochronological analysis. We analyzed the growth pattern of the shells, calculated maximum and minimum increments and compared the results with data from modern shells analyzed by other researchers. The results showed that the highest percentage $(42,30 \%)$ of shellfish were collected in summer. In winter, the percentage of shells collected was 30,77\% and lower values were observed in autumn (15,28\%) and spring (11,54\%). This would indicate, first, resource utilization throughout the year and also a variation in the intensity of harvesting activity on the season.
\end{abstract}

KEY WORDS: Mytilus spp., zooarchaeology, sclerochrology, Patagonia.

\section{INTRODUCCIÓN}

Determinar la estacionalidad del uso humano de las costas en el pasado es un tema que interesa a los arqueólogos. Con este propósito, desde fines de 1970 se vienen aplicando diferentes técnicas de análisis, siendo el estudio de los patrones de crecimiento en los moluscos uno de los métodos más utilizados para determinar la estacionalidad en contextos arqueológicos. (Coutts 1970; Clark II 1979; Rhoads y Lutz 1980; Aten 1982; Claassen 1982, 1986, 1990; Deith 1983, Quitmyer et al. 1997; Andrus 2011; Colonese et al.2011, 2012).

Los moluscos presentan un crecimiento regulado por factores endógenos (edad, reproducción) y exógenos (temperatura, mareas, salinidad, tormentas). Las variaciones de estos factores se ven reflejadas en sus tejidos duros (las valvas) por una modificación en su estructura y composición química (proporciones de carbonato de calcio y materia orgánica) (Gröcke y Gillikin 2008). De esta manera, las líneas de crecimiento conservan en su secuencia de formación un patrón de crecimiento que al ser analizado brinda información sobre las características del ambiente donde vivieron, la perio- dicidad de crecimiento, la influencia de las mareas y la estacionalidad de muerte entre otras cosas (Dodd y Stanton 1981; Jones 1983; Deith 1986; Jones et al. 1990; Jones y Quitmyer 1996; Shöne et al. 2004; Surge y Walker 2005; Soldati 2009).

Uno de los métodos para analizar las marcas de crecimiento es la esclerocronología. La esclerocronología es una técnica que analiza las variaciones físicas y químicas de la acreción de los tejidos duros de los moluscos (Jones et al. 2007), permitiendo establecer, entre otras cosas, la época del año en la que mueren los individuos. Poder determinar ese momento convierte a la esclerocronología en una técnica de gran provecho para la zooarqueología, brindando información para temas de importancia como los patrones de ocupación del espacio, las estrategias de subsistencia de poblaciones humanas y los procesos de formación de sitios, entre otros. (Claassen 1986; Deith y Shackleton 1988; Waselkov 1987; Andrus 2011).

El objetivo de este trabajo consiste en determinar la estacionalidad de recolección de los mejillones Mytilus spp. provenientes del sitio $\mathrm{CCH} 4$, Parque Nacional Monte León y resaltar la importancia que puede tener esta técnica para el estudio del 


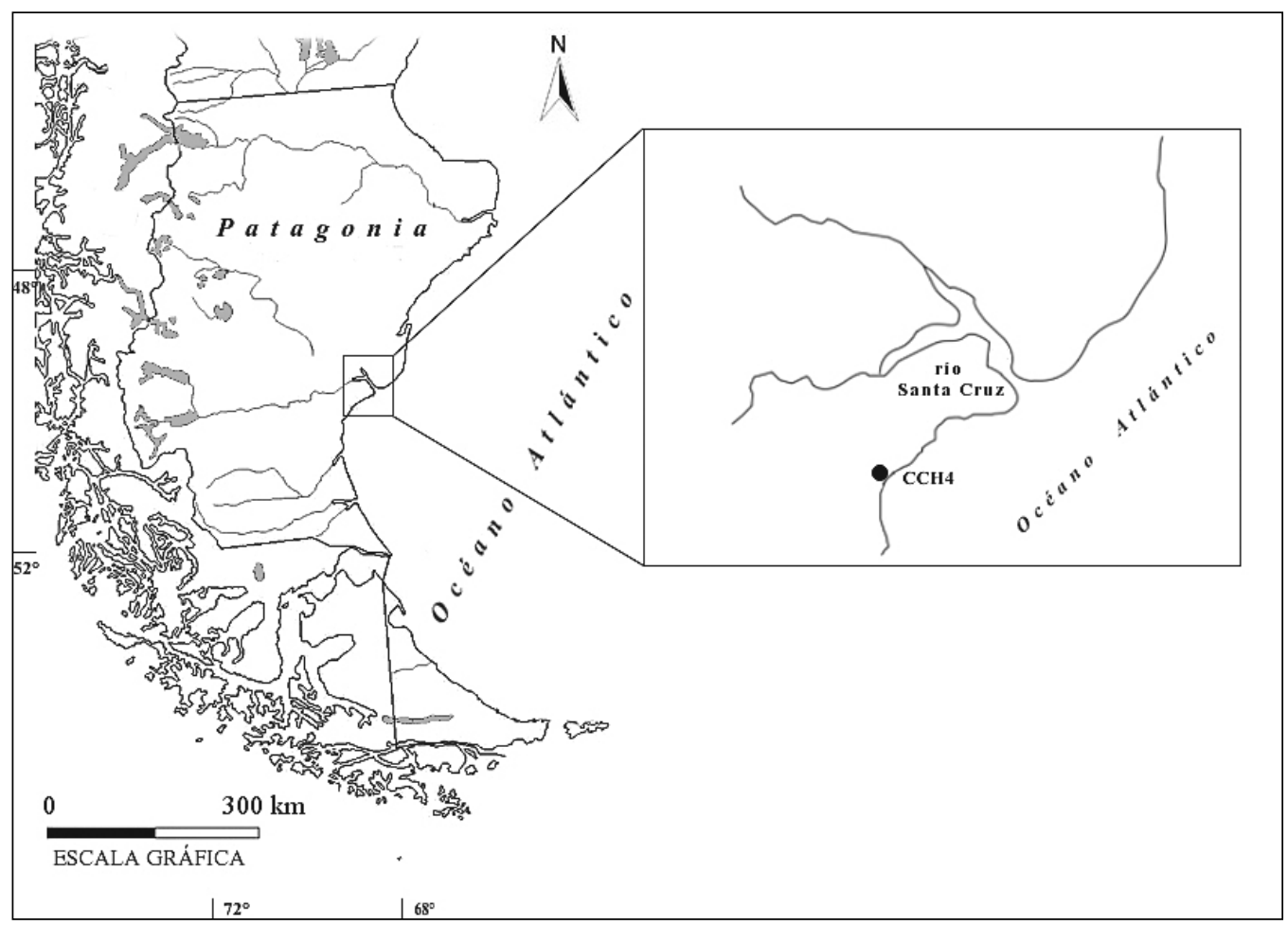

Fig. 1. Ubicación del sitio CCH4, Parque Nacional Monte León (Santa Cruz, Argentina).

poblamiento y uso humano de la costa en el sur del continente. En la costa atlántica de Patagonia, los restos malacológicos constituyen una parte abundante del registro arqueológico. Estos depósitos brindan información acerca de la ocupación humana del litoral atlántico mostrando que constituyeron un recurso que fue explotado al menos desde hace 7.400 años AP. (Zubimendi 2007; entre otros). Por tal motivo, resulta esencial comprender la forma en que las poblaciones humanas utilizaron los diferentes hábitats costeros. Considerando que los moluscos constituyen un recurso disponible todo el año, es fundamental determinar la estación en la que fueron recolectados, no sólo para dilucidar la importancia del recurso en la economía de los grupos humanos involucrados sino también porque ofrecen información valiosa para el análisis de los patrones ocupación humana en la costa.

\section{MATERIAL DE ESTUDIO}

El sitio Conchero $4(\mathrm{CCH} 4)$ se encuentra ubicado en la Pingüinera Norte, al borde de una barranca, en una antigua zona de nidificación de pingüinos de Magallanes (Spheniscus magellanicus), en el Parque Nacional Monte León (Fig. 1). El Parque Nacional Monte León se sitúa al sudeste de la provincia de Santa Cruz, en la costa Argentina. Con una longitud de $40 \mathrm{~km}$, el área costera se caracteriza por estar dominada por sistemas macromareales y presentar una variedad de ambientes intermareales (altos acantilados, playas arenosas, playas de cantos rodados, restingas planas y labradas) que albergan una gran diversidad de recursos marinos, encontrándose grandes bancos de moluscos con especies pertenecientes a la provincia biogeográfica Magallánica (Del Río 2004; Bortolus et al. 2006). Estudios realizados en el Parque han revelado la presencia de sitios arqueológico, observándose concentraciones con abundante material arqueológico como artefactos líticos, fauna terrestre y marina en diferentes lugares de la costa (Caracotche 2003; Castro et al. 2004; Caracotche et al. 2005 y 2008; Muñoz et al. 2009; Cruz et al. 2010.).

Durante los trabajos de campo efectuados en el marco del Proyecto de Rescate Arqueológico Monte León durante abril de 2004 (Caracotche et al. 2005) se recolectaron valvas arqueológicas de Mytilus 
spp.; del sitio Conchero 4 (CCH4). El sitio presenta una concentración de materiales arqueológicos a lo largo de 20,4 m, de la cual se excavó una cuadricula de $0,8 \times 0,5 \mathrm{~m}$, siguiéndose niveles artificiales de $5 \mathrm{~cm}$ y llegándose a una profundidad de $1,29 \mathrm{~m}$ (Caracotche et al. 2005, 2008 y Cruz et al. 2011). Durante la excavación se observaron distintas lentes de valvas y de carbón asociadas a artefactos líticos. Entre los restos arqueofaunísticos recuperados se incluyen mamíferos terrestres (Lama guanicoe) y marinos (pinnípedos y cetáceos), aves (Phalacrocorax sp.), valvas (Aulacomya ater y Mytilus chilensis) y conchillas (Patinigera magallánica, Trophon sp. y Acantina sp.) de moluscos. Estos restos indican el predominio de diversos recursos marinos en la subsistencia humana, reflejando la importancia de la explotación de la costa durante los últimos 2.000 años AP (Cruz et al. 2011).

Para el análisis esclerocronológico se trabajó con muestras provenientes de 4 niveles diferentes (Tabla 1). Se seleccionaron las 27 valvas que presentaron mejor estado de conservación y de mayor calidad en su margen ventral, es decir, un margen ventral sin fracturas ni desgaste en su borde.

\section{METODOLOGÍA}

Las muestras fueron procesadas en el Institut für Geowissenschaften de la Universidad de Johannes Gutenberg de Mainz, Alemania. Para determinar la estacionalidad de muerte de los moluscos se analizaron las líneas de crecimiento por medio de la esclerocronología. Para ello se realizó la limpieza de las valvas, siguiendo los procedimientos de Dunca y Mutvei (2001), para su posterior montaje en tacos y cobertura de las mismas con resina metálica tipo epoxi bifenol-A (WIKO, Greussenheim, Alemania). Las valvas fueron cortadas en forma perpendicular a las líneas de crecimiento, desde el umbo hasta el margen ventral a lo largo del eje de mayor crecimiento con sierra Isomet 1000 (Buehler, Illinois, USA) de baja velocidad (Fig. 2 a). Se realizaron cortes de $3 \mathrm{~mm}$ de espesor que, posteriormente, fueron enrasados en una superficie de vidrio con polvos de $\mathrm{SiC}$ de distintos tamaños (800 y 1200) y pulidas con $1 \mathrm{~mm}$ $\mathrm{Al}_{2} \mathrm{O}_{3}$. Finalmente las muestras fueron teñidas con solución Mutvei por 25 minutos a $38^{\circ} \mathrm{C}$ (Schöne et al. 2005) y luego lavadas con agua desmineralizada y secadas al aire. Antes y después del tratamiento químico, se tomaron fotografías digitales de las muestras con microscopio binocular bajo luz reflejada. El tratamiento con solución Mutvei permite una clara visualización de los patrones y las líneas de crecimiento. Esto es posible debido a que el ácido acético de la solución disuelve el carbonato de calcio mientras que las partes orgánicas son protegidas por gluteraldheidos, durante este proceso los azucares y glicoproteínas de los biominerales son teñidos con Alcian Blue (Schöne et al. 2005).

Para la determinación de la edad se realizó el conteo de las líneas anuales de crecimiento de la capa nacarada siguiendo la metodología de Lutz (1976) y Richardson et al. (1990). Esta metodología constituye una alternativa a la determinación de la edad a partir de las líneas de crecimiento formadas en la superficie externa de la valva.

Para el análisis de los incrementos en el crecimiento de las valvas y de estacionalidad de muerte se procesaron las microfotografías con el programa Panopea (CPeinl y Schöne 2004) a través del cual se realizó un recuento de los incrementos, midiéndose el ancho de los mismos. El ancho del incremento es la distancia entre dos líneas de crecimiento adyacentes (Fig. 6b).

La estacionalidad de muerte se determinó siguiendo la metodología propuesta por Rhoads y Lutz (1980) y Kennish (1980). Se identifico la última marca anual y a partir de ahí se contabilizaron y midieron las líneas de crecimiento hasta el margen ventral. Debido a que en este trabajo no se analizan

Tabla 1. Niveles de excavación y fechados radiocarbónicos de CCH4 (Parque Nacional Monte León), Santa Cruz, Argentina.

\begin{tabular}{|c|c|c|c|c|}
\hline Sitio & Nivel estratigráfico & № Muestras & Edad radiocarbónica (años AP) & Referencia \\
\hline \multirow{5}{*}{$\mathrm{CCH} 4$} & $1(40-48 \mathrm{~cm})$ & 6 & $\begin{array}{c}1330 \pm 60 \mathrm{AP} \\
(\mathrm{LP} \pm 1515)\end{array}$ & Caracotche et al. 2005 \\
\cline { 2 - 5 } & $7(68,5-73,5 \mathrm{~cm})$ & 9 & - & - \\
\cline { 2 - 5 } & $10(83,5-88,5 \mathrm{~cm})$ & 6 & $\begin{array}{c}0 \pm 70 \mathrm{AP} \\
(\mathrm{LP} \pm 1583)\end{array}$ & Caracotche et al. 2005 \\
\hline
\end{tabular}



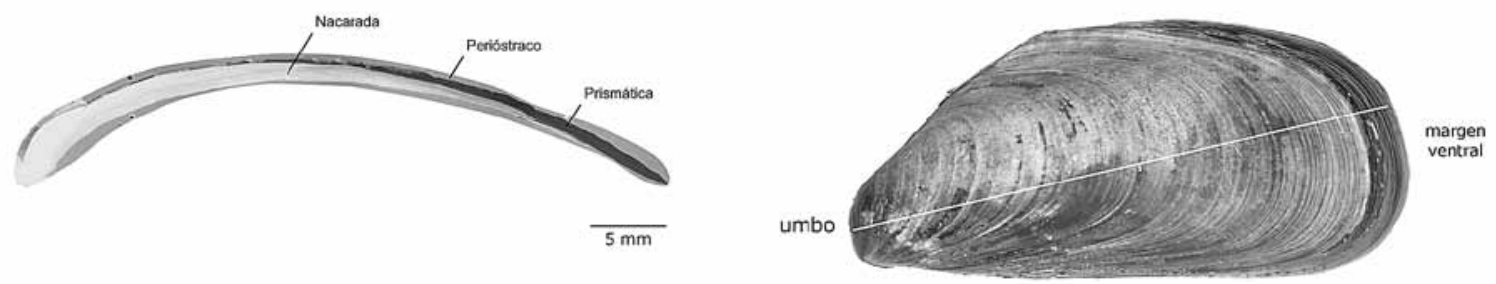

$\overline{5 \mathrm{~mm}}$

Fig. 2. (a) Microfotografía de una sección transversal de Mytilus sp. mostrando la estructura de la valva. (b) Fotografía de la valva izquierda de un ejemplar de Mytilus sp. indicando el eje de crecimiento máximo.

muestras actuales de mejillones, para corroborar los patrones de crecimiento observados en las valvas arqueológicas, se compararon los patrones de crecimiento obtenidos por Richardson (1990) para individuos modernos de Mytilus edulis de las costas de California (Estados Unidos) y por Gray et al. (1997) para Mytilus edulis chilensis de las Islas Malvinas.

Los datos obtenidos fueron procesados a través del programa estadístico SPSS 15.0 (SPSS Inc.). Se obtuvieron estadísticos descriptivos y se analizaron las diferencias de medias según el test $\mathrm{H}$ de Kruskal Wallis. Este test es no paramétrico y se utilizó debido a que los datos no cumplieron con los supuestos para aplicar pruebas paramétricas (prueba de normalidad Shapiro-Wilks y test de Levene para homogeneidad de varianza; $\mathrm{p}<0.01$ )

\section{RESULTADOS}

Las valvas de Mytilus sp. están compuestas por tres capas (Fig. 2 a). La más externa en contacto directo con el medio ambiente, se denomina perióstraco. Le sigue una capa intermedia, la capa prismática, y por último encontramos la capa nacarada en la que se puede observar un patrón bien desarrollado de bandas claras y oscuras intercaladas.

En los cortes transversales, anteriores al teñido con solución Mutvei, en la capa prismática se pueden observar, los incrementos periódicos como una alternancia de líneas claras y oscuras (Fig. 3a). Luego del tratamiento con solución Mutvei, las líneas oscuras se tiñen de un color azul oscuro mientras que las bandas claras toman un color azul claro, diferenciando ambas claramente (Fig. 3b).

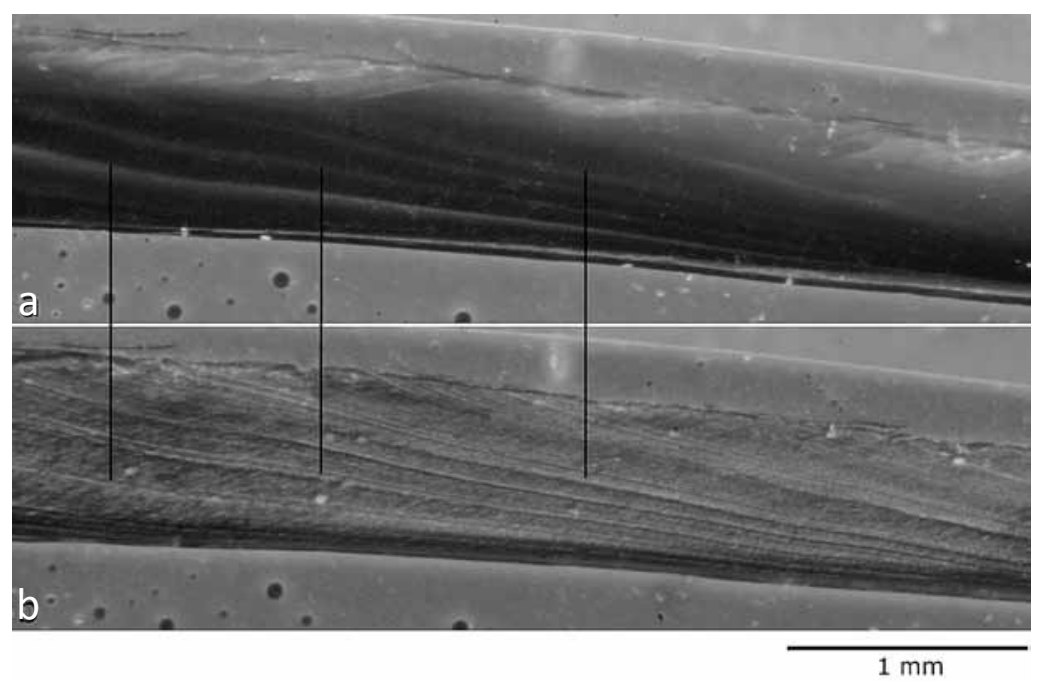

Fig. 3. (a) Mytilus sp. Microfotografía de un corte transversal antes de la tinción (b) posterior al tratamiento con solución Mutvei. 
a

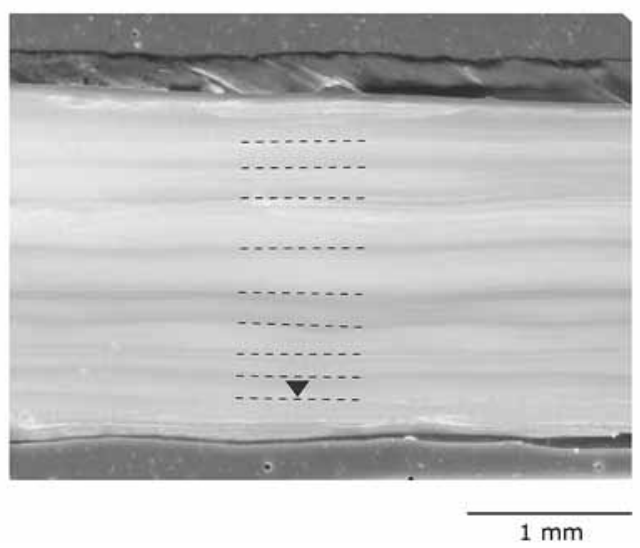

Fig.4. Mytilus sp. Microfotografía de una sección transversal donde se puede observar en detalle las nueve líneas de crecimiento (líneas punteadas) desarrolladas sobre la capa nacarada.

La edad de los individuos (Fig. 4a), determinada contando las líneas de crecimiento anual observadas en la capa nacarada, indicó un rango de edad de 4 a 10 años, presentando en promedio incrementos diarios de $(0,0017 \pm 0,0005) \mathrm{cm}$.

El análisis de las líneas de crecimiento observada en Mytilus spp. refleja un conjunto de 6-8 líneas de crecimiento claras y nítidas, seguidas de otro grupo de 6-8 líneas difusas. Esto estaría indi- cando un crecimiento denominando quincenal o fortnightly, en donde cada grupo de líneas corresponderían a la influencia de las mareas vivas (ocurre durante luna nueva y llena, siendo la amplitud de las mareas máximas) y las mareas muertas (ocurre durante cuarto creciente y cuarto menguante siendo la amplitud de las mareas mínima). Las líneas formadas durante las mareas vivas son más nítidas y definidas que aquellas formadas durante las mareas muertas (Fig. 5). Este mismo patrón de crecimiento quincenal fue el observado por Richardson et al. (1990) y por Gray et al. (1997). Las mediciones del crecimiento desde el comienzo de la marea viva hasta la marea muerta refleja un aumento periódico promedio de $(0,02 \pm 0,005) \mathrm{cm}$ cada 14 días. Este valor es menor al obtenido por Richardson et al. (1990), quienes observaron un valor mínimo de $0,03 \mathrm{~mm}$ y un máximo de 0,2 $\mathrm{mm}$, sin embargo estas diferencias pueden deberse a características ambientales (temperatura, latitud, concentración de oxígeno, etc.) de cada zona.

Con el fin de establecer la estacionalidad se analizaron las líneas de crecimiento observadas a partir de la última marca anual hasta el margen ventral. En la Fig. 6 se puede observar el patrón de crecimiento desde el otoño/invierno hasta el verano. Durante el otoño el crecimiento comienza a disminuir hasta llegar al mínimo crecimiento en

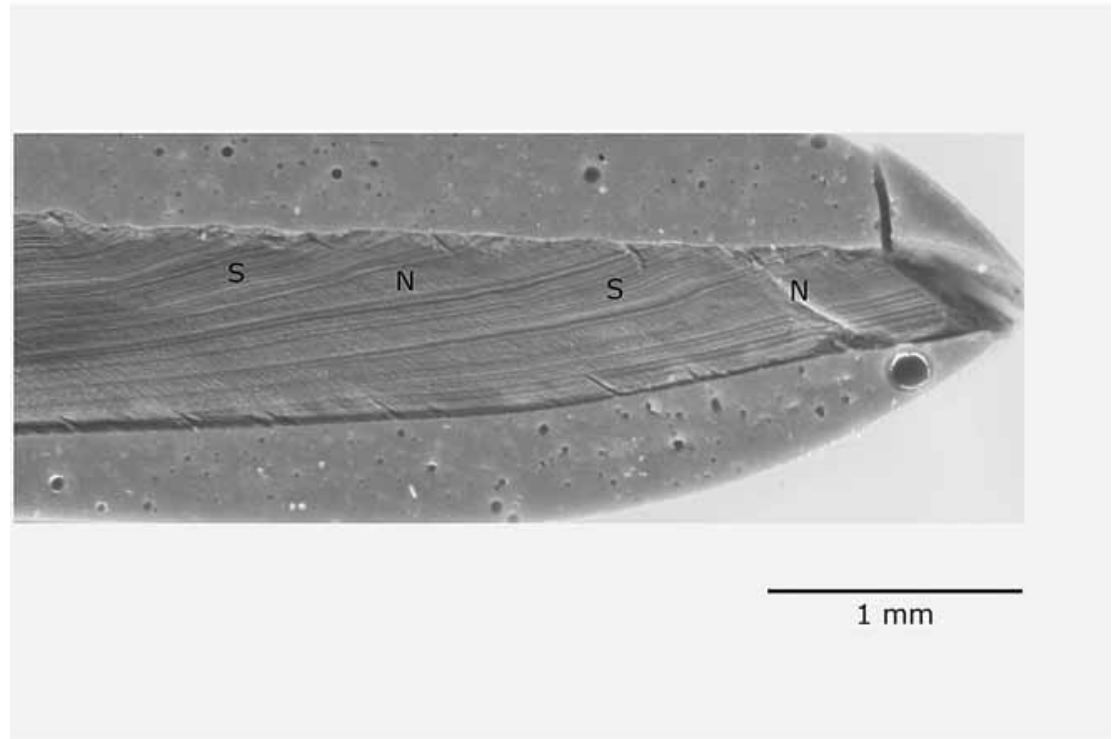

Fig. 5. Mytilus sp. Sección transversal donde se pueden observar los ciclos de mareas vivas (S) y muertas (N). Durante la marea viva se forman 6 a 8 líneas nítidas y durante las mareas muertas entre 6-8 líneas borrosas. 
a

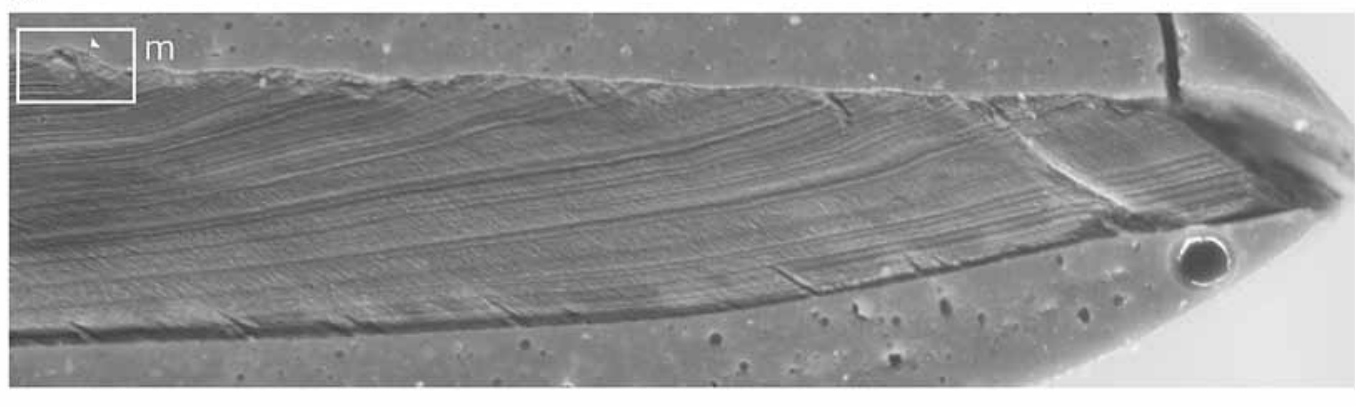

b

$1 \mathrm{~mm}$
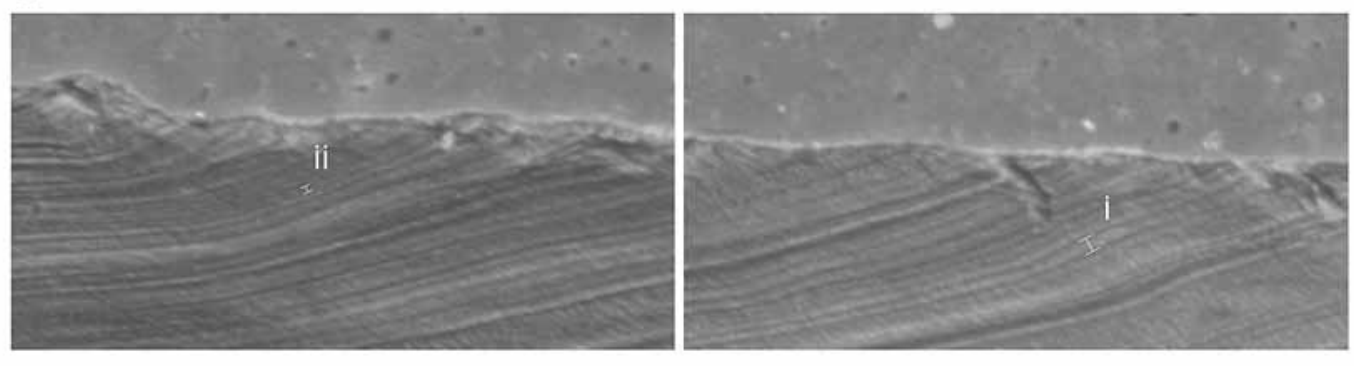

$300 \mu$

Fig. 6. (a) (b)Mytilus sp (m: marca anual) (b) ii: incrementos durante el invierno, i: incremento durante el verano

invierno, observándose la menor distancia entre dos líneas consecutivas. Además durante ésta última estación las líneas de crecimiento convergen en el perióstraco formando una marca anual (Fig. 6a). Durante la primavera y el verano el crecimiento es más rápido, presentándose una mayor distancia entre dos líneas consecutivas (Fig. 6b). Al comparar el ancho de los incrementos en cada una de las estaciones se observaron diferencias significativas $(p<0,001)$.

Al determinar la estacionalidad de muerte de los mejillones de $\mathrm{CCH} 4$, se encontró que el mayor porcentaje $(42,30 \%)$ fueron recolectados en verano. En invierno se colectó el 30,77\% de estos invertebrados y valores menores fueron observados en otoño $(15,28 \%)$ y primavera (11,54\%). En la Figura 7 se puede observar el porcentaje de cada estación por nivel de excavación. El nivel 1 presenta una colecta centrada en las estaciones cálidas (primavera y verano) a diferencia del nivel 7 que, por lo contrario, presenta estaciones más frías como son otoño $e$ invierno. En el nivel 10 no se observa recolección en primavera y el nivel 11 tiene representadas todas las estaciones.

\section{DISCUSIÓN}

Los resultados obtenidos muestran que la esclerocronología permite un claro análisis de las líneas de crecimiento de estos moluscos. A pesar de tratarse de individuos arqueológicos sometidos a distintos procesos tafonómicos que afectan su integridad estructural, es posible visualizar de manera clara, luego del tratamiento con solución Mutvei, todas las líneas de crecimiento.

El patrón de crecimiento observado en las valvas de $\mathrm{CCH} 4$ presentó una periodicidad de 14 días. Esto también fue observado por Richardson (1989), Richardson et al. (1990) y Gray et al. (1997) en individuos modernos de Mytilus edulis. Durante las mareas vivas las líneas de crecimiento presentan mayor nitidez diferenciándose de las formadas durante las mareas muertas que son débiles y más difíciles de observar. Estudios realizados por Lutz y Rhoads (1977) y Gordon y Carriker (1978) indican que esta variación observada durante las mareas vivas (cuando los moluscos deben cerrar sus valvas al ser expuestos al aire dos veces al día) y mareas 


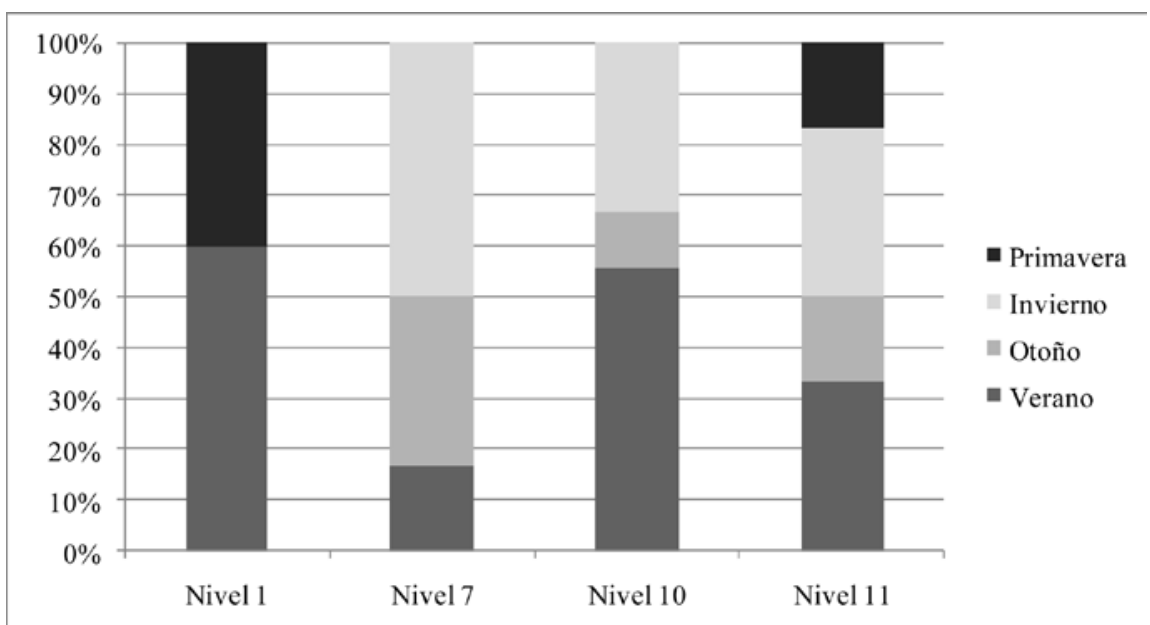

Fig. 7. Porcentajes de individuos pertenecientes a cada estación por nivel de excavación en CCH 4 (Parque Nacional Monte León).

muertas (cuando pasan la mayor parte del tiempo sumergidos), es producto de una alternancia de depositación y disolución durante los procesos anaeróbicos. Mientras los moluscos están sumergidos se deposita carbonato de calcio $\left(\mathrm{CaO}_{3}\right)$ y materia orgánica, pero cuando están expuestas al aire cierran sus valvas y comienza el metabolismo anaeróbico a partir del cual se disuelve el carbonato de calcio y se forman líneas ricas en materia orgánica (Crenshaw y Neff 1969; Kennish 1980).

Si bien trabajar con valvas arqueológicas no presenta dificultades para el análisis de las líneas de crecimiento de la capa prismática, no ocurre lo mismo al analizar las líneas de la capa externa. Los diversos factores erosivos a los que están expuestas las valvas afectan su superficie externa, la cual presenta distintos estados de desgaste por lo que es imposible determinar de la edad, a partir de esta superficie. Sin embargo la determinación de la edad de muerte a partir de las líneas anuales en la superficie nacarada resulta una técnica alternativa y viable. Esto mismo fue observado por Richardson et al. (1990), Lutz (1976), Bayne y Worrall (1980) y Rodhouse et al. (1984), quienes determinaron la edad de los individuos a partir de la capa nacarada y obtuvieron una correlación significativa al comparar esta edad con la determinada a partir de la superficie externa.

Con respecto a la estacionalidad de recolección en $\mathrm{CCH} \mathrm{4,} \mathrm{los} \mathrm{resultados} \mathrm{indicarían} \mathrm{que}$ la actividad no estaría centrada en una sola esta- ción. Los mayores porcentajes de recolección se observaron en las estaciones de invierno y verano, disminuyendo en otoño y primavera. Esto estaría indicando, por un lado, una utilización del recurso durante todo el año y también una variación en la intensidad de la actividad de recolección de acuerdo a la estación. Estos resultados no coinciden con lo observado por Moreno (2008) quien, por medio del análisis de restos faunísticos de mamíferos y aves, entre otros, concluye que la mayor frecuencia de uso de la costa ocurre durante los meses cálidos del año (desde septiembre hasta mayo). Esta diferencia en los resultados podría deberse a que la disponibilidad y accesibilidad varía entre los distintos recursos y a que, además, la dieta de los grupos humanos está constituida por diferentes recursos alimenticios, lo cual significa que determinar la estacionalidad de adquisición de un solo recurso brinda información muy importante pero no concluyente sobre los patrones de ocupación de un determinado sector de la costa. A esto debemos sumarle que los resultados obtenidos a partir de los recursos faunísticos tampoco concuerdan con los resultados isotópicos. Moreno et al. (2011) observan una preponderancia de dietas mixtas, lo que indica una ingesta combinada de recursos marinos y terrestres. En cambio, Suby et al. (2009) señalan un consumo muy bajo de alimentos marinos para cuatro individuos provenientes de la cuenca inferior del río Santa Cruz y zonas aledañas, es decir, en el área de estudio de este trabajo. De esta manera, a 
partir de los resultados isotópicos y arqueológicos, tal como fuera planteado por Cruz et al. (2011), no puede asociarse el uso de la costa en sectores aledaños a Monte León con una estación específica, sugiriéndose un uso a intervalos variables en el tiempo, lo cual plantea la necesidad de ampliar la escala de los análisis arqueológicos e isotópicos.

\section{CONSIDERACIONES FINALES}

Dado que varios modelos sobre el poblamiento y uso humano de la costa en el sur del continente plantean un uso estacional de la misma, la esclerocronología aparece como una herramienta fundamental $e$ independiente para discutirlos. Esta técnica aporta una nueva línea de evidencia zooarqueológica a esta discusión, la cual sumada a otras, permitirá estimar la estacionalidad de las ocupaciones humanas en sectores específicos de la costa patagónica.

Es necesario señalar que, si bien la esclerocronología es una buena técnica para el análisis de estacionalidad de moluscos arqueológicos, resulta necesario realizar de manera paralela estudios esclerocronológicos en individuos modernos de la misma zona de estudio. Al tener un control con individuos modernos mejoraría la calidad de los resultados arqueológicos y la fidelidad de los mismos.

Finalmente es importante destacar que esta técnica no solo permite determinar la estacionalidad de muerte de los moluscos, sino que brinda información ecológica, paleoambiental y del clima del pasado permitiendo un análisis más completo del comportamiento humano, de la formación de los depósitos, de la utilización de los recursos y los sitios.

\section{AGRADECIMIENTOS}

Se agredece a la Dras. María M. Bianchi, Dorrit E. Jacob y Analía L. Soldati por su formación en la preparación de las muestras, asistencia en el laboratorio y la bibliografía brindada y al Dr. Benrd $\mathrm{R}$. Shöne por poner a disposición las instalaciones para el procesamiento de las muestras. Se agradece así mismo los aportes de la Dra. Isabel Cruz y el Dr. A. Sebastián Muñoz, que han contribuido a mejorar considerablemente el artículo. Este trabajo fue realizado gracias a una cooperación bilateral germano/argentina y fue financiado por el Ministerio de Ciencia y Tecnología de Argentina (MINCyT) ARG
07/007, la BMBF alemana y también por UBACYT 447, PIP 112200801 00996, SECYT-UNC 05/ F723 y UNPA 29/A260-1. Agradezco asimismo el apoyo brindado por la Administración de Parques Nacionales y el Parque Nacional Monte León.

\section{BIBLIOGRAFÍA}

ANDRUS, C. F. T. 2011. Shell midden sclerochronogy. Quaternary Science Reviews 30: 2892-2905.

ATEN, L. E. 1982. Determining seasonality of Rangia cuneata from Gulf Coast shell middens. Bulletin of the Texas Archaeological Society 52: 179-200.

BAYNE, B. L. y C. M. WORRALL. 1980. Growth and production of mussels Mytilus edulis from two populations. Mar Ecol.Prog. Ser 3: 317-328.

BORTOLUS, A.; E. SCHWINDT; M. M. MENDEZ; N. ORTIZ; M. E. RÉ; M. L. PIRIZ; I. ELIAS; A. E. GOSZTONYI; L. KUBA; N. CIOCCO; D. A. GAGLIARDINI y J. M. ORENSANZ. 2006. Informe Técnico Final "Estudio de línea de base y plan de monitoreo de la biodiversidad de los ambientes marinos adyacentes al Parque Nacional Monte León". En el marco del proyecto de Conservación de la Biodiversidad. UEP Donación BIRF / GEF TF 028372-A

CARACOTCHE, M. S. 2003. La Arqueología de Monte León: Informe de situación. Delegación Regional Patagonia. Administración de Parques Nacionales MS.

CARACOTCHE, M. S.; F. CARBALLO MARINA; J. B. BELARDI; I. CRUZ y S. ESPINOSA. 2008. Parque Nacional Monte León (Santa Cruz): un enfoque desde la conservación. En: Arqueología de la costa patagónica. Perspectivas para la conservación. Editado por I. Cruz. y M. S. Caracotche, pp. 147-159. Universidad Nacional de la Patagonia Austral - Subsecretaría de Cultura de la Provincia de Santa Cruz

CARACOTCHE, M. S.; I. CRUZ; S. ESPINOSA; F. CARBALLO y J. BELARDI. 2005. Rescate arqueológico en el Parque Nacional Monte León (Santa Cruz, Argentina). Magallania 33(2):143-163.

CASTRO, A.; J. GOMEZ OTERO; G.. ARRIGONI y J. E. MORENO. 2004. Prospección macrorregional comparativa a las Loberías de la costa de la Costa Atlántica Continental de Patagonia: algunas claves para el uso del espacio y de otros recursos. En: Contra viento y marea. Arqueología de la Patagonia. Editado por T. Civalero, P. Fernández y A.G. Guraieb. Instituto Nacional de Antropología y Pensamiento Latinoamericano, Buenos Aires, pp. 197-215 
CLAASSEN, C. P. 1982. Shellfishing patterns: An Analytical Study of Prehistoric Shell from North Carolina Coastal Middens. Ph.D. Dissertation, Harvard University, Cambridge, MA.

CLAASSEN, C. P. 1986. Shellfishing seasons in the prehistoric Southeastern United States. American Antiquity 51: 21-37.

CLAASSEN, C. P. 1990. Investigation of Monthly Growth in Shellfish for Application to Archaeology. Final Report, National Science Foundation Grant BNS-8507714, 129 pp.

CLARK, G. R. II. 1979. Seasonal growth variations in the shells of recent and prehistoric specimens of Mercenaria mercenaria from St. Catherines Island, Georgia. Anthropological Papers of the American Museum of Natural History 51: 161-179.

COLONESE, A.; E. CAMARÓS, E. VERDÚN, J. ESTÉVEZ, S. GIRALT y M. REJAS. 2011. Integrated archaeozoological research of Shell middens: new insights into huntergathered-fisher coastal exploitation in Tierra del Fuego. Journal of Island y Coastal Archaeology, 6: 235-254.

COLONESE, A.; E. VERDÚN; M. ÁLVAREZ, B. ZURRO y L. SALVATELLI. 2012. Oxygen isotopic composition of limpet shells from the Beagle Channel: implications for seasonal studies in Shell middens of Tierra del Fuego. Journal of Archaeological Science, 39: 1738-1748.

COUTTS, P. J. F. 1970. Bivalve growth patterning as a method for seasonal dating in archaeology. Nature 226: 874.

CRENSHAW, M. A. y J. M. NEFF. 1969. Decalcification at the Mantle-Shell Interface in Molluscs. Amer. Zool., 9 (3): 881-885.

CRUZ, I.; A. S, MUÑOZ y P. A. LOBBIA. 2011. La explotación de recursos marinos en la costa de Patagonia continental: Análisis de casos de Punta Entrada y Monte León (Santa Cruz, Patagonia Argentina). Revista de Estudios Marítimos y Sociales (en prensa).

CRUZ, I.; A. S. MUÑOZ y P. A. LOBBIA. 2010. Zooarqueología al sur del río Santa Cruz (Patagonia Argentina). En: Arqueología Argentina en el Bicentenario de la Revolución de Mayo. Editado por: J. Bárcena y H. Chiavazza, Mendoza, Universidad Nacional de Cuyo CONICET, Tomo I, pp. 315-320.

DEITH, M. R. 1983. Molluscan calendars: the use of growth-line analysis to establish seasonality of shellfish collection at the Mesolithic site of Morton, Fife. Journal of Archaeological Science 10: 423-440.

DEITH, M. R. 1986. Subsistence strategies at a Mesolithic camp site: evidence from stable isotope analysis of shells. Journal of Archaeological Science 13: 61-78.
DEITH, M. R. y N. J. SHACKLETON. 1988. Oxygen isotope analyses of marine molluscs from Franchthi Cave. En: Shackleton, J.C. (Ed.), Marine Molluscan Remains from Franchthi Cave. 133-156. Indiana University Press, Bloomington/Indianapolis.

DEL RÍO, C. J. 2004. Relaciones biogeográficas entre los Moluscos del Mioceno Tardío y Reciente del Atlántico Sudoccidental. Miscelánea 12: 39-44.

DODD, R. J. y R. J. JR. STANTON. 1981. Paleoecology, concepts and applicantions. John Wiley and Sons, New York. 559pp

DUNCA, E. y H. MUTVEI. 2001. Comparison of microgrowth pattern in Margaritifera margaritifera shells from south and north Sweden. Am. Malacol. Bull. 16: 239- 250.

GORDON, J. y M. R. CARRIKER. 1978. Growth Lines in a Bivalve Mollusk: Subdaily Patterns and Dissolution of the Shell. Science, 202 (4367): 519-521.

GRAY A. P., R. SEED y C. A. RICHARDSON. 1997. Reproduction and growth of Mytilus edulis chilensis from the Falkland Islands. Scientia Marina, 61 (2): 39-48.

GRÖCKE, D. R. y D. P. GILLIKIN. 2008. Advances in mollusc sclerochronology and sclerochemistry: tools for understanding climate and environment. Geo-Marine Letters, 28: 265-268.

JONES, D. S. 1983. Sclerochronology: reading the record of the molluscan shell. American Scientist, 71: 384-391.

JONES, D. S.; B. ARNOLD; I. R. QUITMYER; B. R. SCHÖNE y D. SURGE. 2007. 1st International Sclerochronology Conference. http://conference.fas.ufl.edu/sclerochronology. Accessed 28 April 2008

JONES, D. S.; I. R QUITMYER; W. S. ARNOLD y D. C. MARELLI. 1990. Annual shell banding, age, and growth rate of hard clams (Mercenaria spp.) from Florida. Journal of Shellfish Research, 9: 215-225

JONES, D.S. y I. R. QUITMYER. 1996. Marking time with bivalve shells: oxygen isotopes and season of annual increment formation. Palaios, 11: 340-346.

KENNISH, M. J. 1980. Shell microgrowth analysis: Mercenaria mercenaria (Linne') as a type example for research in population dynamics. En: Skeletal Growth of Aquatic Organisms. Editado por D.C. Rhoads y R. A. Lutz. Plenum Publishing Corp., New York ,pp.255-294.

LUTZ, R. A. 1976. Annual growth patterns in the inner shell layer of Mytilus edulis L. J. Mar. Biol. Ass. 56: 723-731.

LUTZ, R. A. y D. C. RHOADS. 1977. Anaerobiosis and a theory of growth line formation. Science 198: 1222-1227.

MORENO, E. 2008. Arqueología y etnohistoria de la Costa Patagónica Central en el Holoceno Tardío. Fondo Editorial de la Provincia del Chubut, Rawson. 
MORENO, E.; A. F. ZANGRANDO; A. TESSONE; A. CASTRO y H. PANARELLO. 2011. Isótopos estables, fauna y tecnología en el estudio de los cazadores-recolectores de la costa norte de Santa Cruz. Magallania 39 (1): 265-276.

MUÑOZ, S. A; M. S., CARACOTCHE e I. CRUZ. 2009. Cronología de la costa al sur del río Santa Cruz: nuevas dataciones en Punta Entrada y Parque Nacional Monte León (Provincia de Santa Cruz, Argentina). Magallania 37, (1): 39-43.

QUITMYER, I. R.; D. S. JONES y W. S. ARNOLD. 1997. The Sclerochronolgy of Hard Clams, Mercenaria spp., from the South-Eastern U.S.A.: A Method of Elucidating the Zooarchaeological Records of Seasonal Resource Procurement and Seasonality in Prehistoric Shell Middens. Journal of Archaeological Science 24(9):825-840.

RHOADS, D. C.y R. A. LUTZ, (Eds). 1980. Skeletal Growth of Aquatic Organisms. Plenum Press, New York.

RICHARDSON, C.A. 1989. An analysis of the microgrowth bands in the shell of the common mussel Mytilus edulis. J. Mar. Biol. Ass. U. K., 69: 477-491.

RICHARDSON, C.A.; R. SEED y E. NAYLOR. 1990. Use of internal growth bands for measuring individual and population growth rates in Mytilus edulis from offshore production platforms. Mar. Ecol. Prog. Ser., 66: 259-265.

RODHOUSE, P. G.; C. M. RODEN; G. M. BURNELL.; M. P. HENSEY; T. MCMAHON; B. OTTWAY y T H. RYAN. 1984. Food resource, gametogenesis and growth of Mytilus edulis on the shore and in suspended culture: Killary Harbour. Ireland. J. Mar. Biol. Ass. U.K., 64: 513-529.
SCHÖNE, B.R.; E. DUNCA; H. MUTVEI y U. NORLUND. 2004. A 217- year record of summer air temperature reconstructed from freshwater pearl mussels (M. margarifitera, Sweden).Quaternary Science Reviews, 23: 1803-1816.

SCHÖNE, B.R.; E. DUNCA; J. FIEBIG y M. PFEIFFER. 2005. Mutvei's solution: An ideal agent for resolving microgrowth structures of biogenic carbonates. Palaeogeography, Palaeoclimatology, Palaeoecology 228: 149-166.

SOLDATI, A. L.; D.E. JACOB; B. R. SCHÖNE; M. M. BIANCHI y A. HAJDUK. 2009. Seasonal periodicity of growth and composition in valves of Diplodon chilensis patagonicus (D'Orbigny, 1835). Journal of Molluscan Studies 75: 75-85.

SUBY, J.; R. GUICHÓN y A. ZANGRANDO. 2009. El registro biológico humano de la costa meridional de Santa Cruz. Revista Argentina de Antropología Biológica 11 (1): 109-124.

SURGE, D.y K. J. WALKER. 2005. Oxygen isotope composition of modern and archaeological otoliths from the estuarine hardhead catfish (Ariopsis felis) and their potential to record low-latitude climate change. Palaeogeography, Palaeoclimatology, Palaeoecology 22: 179-191.

WASSELKOV, G. A. 1987. Shellfish gathering and shell midden archaeology. En Advances in Archaeological Method and Theory 10. Editado por: M. B. Schiffer M. B. Academic Press, Nueva York, pp. 93- 210

ZUBIMENDI, M. A. 2007. Discusión sobre las malacofaunas presentes en sitios arqueológicos de la Patagonia Continental Argentina. Publicación digital en el CD de las VI Jornadas de Arqueología e Historia de las Regiones Pampeana y Patagónica. 
